\title{
UTILIZAÇÃO DE BÓRAX POR DIFUSÃO NO TRATAMENTO DE PRESERVAÇÃO DE LÂMINAS DE SUMAÚMA (Ceiba pentantra (L.) Gaertn.) ${ }^{1}$
}

\author{
André Luis Willerding ${ }^{2}$ e Basílio Frasco Vianez ${ }^{3}$
}

\begin{abstract}
RESUMO - O objetivo deste trabalho foi comparar três diferentes intervalos de tempo para difusão do bórax, visando a preservação de lâminas de sumaúma. $\mathrm{O}$ tratamento foi a aplicação por aspersão de $30 \mathrm{ml}$ de solução, a $4 \%$ de concentração de bórax. Na pesquisa, optou-se pela retenção de $0,500 \%$ de equivalente de ácido bórico (EAB), com base na massa das lâminas secas. Avaliou-se a retenção nos tempos de difusão de 15, 150 e 270 minutos. Por meio da análise química quantitativa, foram verificados os níveis de retenção por espectrofotometria, obtendose $0,462,0,457$ e $0,440 \%$ de $E A B$, respectivamente, para os tratamentos de 15,150 e 270 minutos, que não diferiram estatisticamente entre si pelo teste Tukey $(\mathrm{p}<0,05)$. Os resultados indicam uma boa difusão do bórax aos 15 minutos, o que demonstrou ser uma alternativa como tratamento preservante de madeira.
\end{abstract}

Palavras-chave: Bórax, madeira tropical, difusão, Ceiba pentantra, sumaúma e Amazônia.

\section{BORAX DIFFUSION TREATMENT IN THE PRESERVATION OF SUMAUMA (Ceiba pentantra (L.) Gaertn.) VENEER}

\begin{abstract}
The objective of this work was to compare three different time intervals for diffusion treatments using borax in the preservation of sumauma veneer. The treatment consisted of application by aspersion of $30 \mathrm{ml}$ borax solution at 4\% concentration. Retention of $0.500 \%$ of boric acid equivalent (BAE), was chosen, based on dry blade mass. Retention was evaluated in the diffusion times of 15, 150 and 270 minutes. Retention levels were verified by quantitative chemical analysis, through spectrophotometric measurement, with the following levels being obtained: 0.462; 0.457 and 0.440\% BAE, respectively, for the treatments of 15, 150 and 270 minutes, which did not differ significantly by the Tukey test $(p<0.05)$. The results indicate a good borax diffusion at 15 minutes, which was found to be an alternative treatment for timber preservation.
\end{abstract}

Key words: Borax, tropical timber, diffusion, (Ceiba pentantra) sumaúma and Amazon.

\section{INTRODUÇÃO}

A madeira, como recurso natural renovável, deve ser utilizada de forma racional e não ser considerada como matéria-prima inesgotável e de baixo custo. Assim, o desenvolvimento de tecnologias de preservação tem contribuído para aumentar a durabilidade da madeira. $\mathrm{O}$ emprego de produtos como o bórax (tetraborato dissódio decahidratado), em virtude de suas características, tornase atrativo perante a atual consciência ecológica e ambiental, na qual procura-se usar produtos mais seguros para a preservação de madeiras, quando comparados aos

1 Recebido para publicação em 4.4.2001.

Aceito para publicação em 12.5.2003.

2 Pesquisador Bolsista PCI/MCT/INPA. Instituto Nacional de Pesquisas da Amazônia. Coordenação de Pesquisas em Ciências Agronômicas - CPCA. Av. André Araújo, 2936. Bairro Petrópolis. 69083-000 Manaus-AM, <andrelw@inpa.gov.br>; ${ }^{3}$ Pesquisador Titular. Instituto Nacional de Pesquisas da Amazônia. Coordenação de Pesquisas em Produtos Florestais - CPPF, $<$ basilio@inpa.gov.br>. 
produtos convencionais. O bórax caracteriza-se como não tóxico ao meio ambiente, hidrossolúvel e relativamente eficiente contra fungos e insetos xilófagos, além de ser ignífugo e possuir baixa toxicidade a mamíferos (Carr, 1958b; Laks et al., 1988; Amburgey, 1990; Morrel et al., 1990).

O método de difusão, no tratamento da madeira, resulta em economia por causa dos baixos custos de mãode-obra e de equipamentos envolvidos no processo (Carr, 1958a).

Os principais fatores relacionados à difusão na madeira são o teor de umidade, a densidade, as características anatômicas e a espessura da peça a ser tratada, além das características físico-químicas do produto preservante a ser empregado. Destes fatores, o teor de umidade é o principal (Becker, 1976; Cockcroft \& Levy, 1973; Morrel \& Freitag, 1995; Puettmann \& Schmidt, 1997), pois a madeira deve estar úmida para favorecer a difusão das moléculas do preservativo para o interior da peça.

Neste estudo utilizou-se a espécie Ceiba pentandra (L.) Gaertn. (sumaúma), que é caracterizada como madeira leve (densidade básica entre 0,30 e $0,37 \mathrm{~g} / \mathrm{cm}^{3}$ ) e é empregada para embalagens, móveis e celulose, sendo classificada como altamente impregnável (Loureiro et al., 1979; Chudnoff, 1980). No entanto, no Estado do Amazonas, o maior emprego da madeira de sumaúma é na confecção de lâminas para produção de compensado (Hummel et al., 1993).

A madeira de sumaúma é suscetível à podridãobranca e à podridão-parda, a fungos manchadores e a insetos (Chudnoff, 1980; Déon, 1986). Por tais características, notam-se a necessidade e a possibilidade do uso de preservantes. Assim, o objetivo deste trabalho foi comparar três diferentes intervalos de tempo de aplicação de bórax por difusão, visando a preservação de lâminas de sumaúma.

\section{MATERIAL E MÉTODOS}

\subsection{Local e Laminação da Madeira}

O trabalho foi realizado no Setor de Lâminas e Compensados da Coordenação de Pesquisas em Produtos Florestais (CPPF) do Instituto Nacional de Pesquisas da Amazônia (INPA), Manaus, Amazonas, Brasil.

Utilizaram-se três toras de $80 \mathrm{~cm}$ de diâmetro e com os comprimentos variando de 1,7 a $2,0 \mathrm{~m}$, procedentes de três árvores diferentes. Aplicaram-se três diferentes tempos de difusão de 15,150 e 270 minutos, identificados, respectivamente, como tratamentos 1,2 e 3 . Os tempos de tratamento foram estabelecidos em função de um tempo ocioso, constatado em linhas de produção de compensado, em que as lâminas são rebobinadas após o desenrolamento e permanecem ali à espera da guilhotinagem. Tal fato ocorre em virtude da menor produtividade da guilhotina em relação ao torno desenrolador, sendo esta etapa mais lenta na linha de produção do compensado, obtendo-se, no máximo, $45 \%$ da produtividade do torno (Zavala, 1990). Desta forma, há um acúmulo de bobinas na linha de produção, sendo a etapa em que o processo de tratamento de lâminas pode ser inserido na fábrica.

A laminação foi processada em um torno da marca Fezer, modelo 15 PA 18. Para retirada das lâminas das toras, foram determinadas três seções, classificadas como: externa (E), média (M) e interna (I), conforme a Figura 1. Procurou-se, desta forma, minimizar o erro experimental causado pela variação nas características da madeira do alburno e do cerne, além de promover a melhor representatividade nas toras.

Na seqüência, dividiu-se cada tora em três seções com espessuras proporcionais, a fim de compensar a diminuição do volume da peça durante o torneamento, tendo a seção externa $10 \mathrm{~cm}$, a média $20 \mathrm{~cm}$ e a interna $30 \mathrm{~cm}$, havendo ainda um desconto de $15 \mathrm{~cm}$ para o rolo resto e $5 \mathrm{~cm}$ para o acerto do cilindro da tora.

Para a amostragem, selecionou-se uma seção diferente por tora, de onde foram retiradas aleatoriamente 20 lâminas, totalizando 60 lâminas de $0,002 \mathrm{~m}$ de espessura para cada tratamento. Em seguida, as lâminas foram redimensionadas em uma serra circular e ficaram em $0,50 \times 0,50 \mathrm{~m}$, a fim de se tornarem corpos-de-prova.

\subsection{Tratamento das Lâminas}

No tratamento preservativo, os corpos-de-prova foram borrifados em uma face com $30 \mathrm{ml}$ de solução de bórax a $4 \%$ de concentração, cujo ingrediente ativo (IA) é expresso em porcentagem de equivalente de ácido bórico (EAB). O EAB é a relação entre a massa do IA e a massa da madeira seca dos corpos-de-prova. A concentração foi estabelecida pelo fato de que o bórax, nas regiões tropicais, deve ter uma concentração aplicável de até $5 \%$ da solução, pois acima desta ocorre a precipitação dos sais de boro na solução, havendo erros de concentração (Vianez, 1993). 


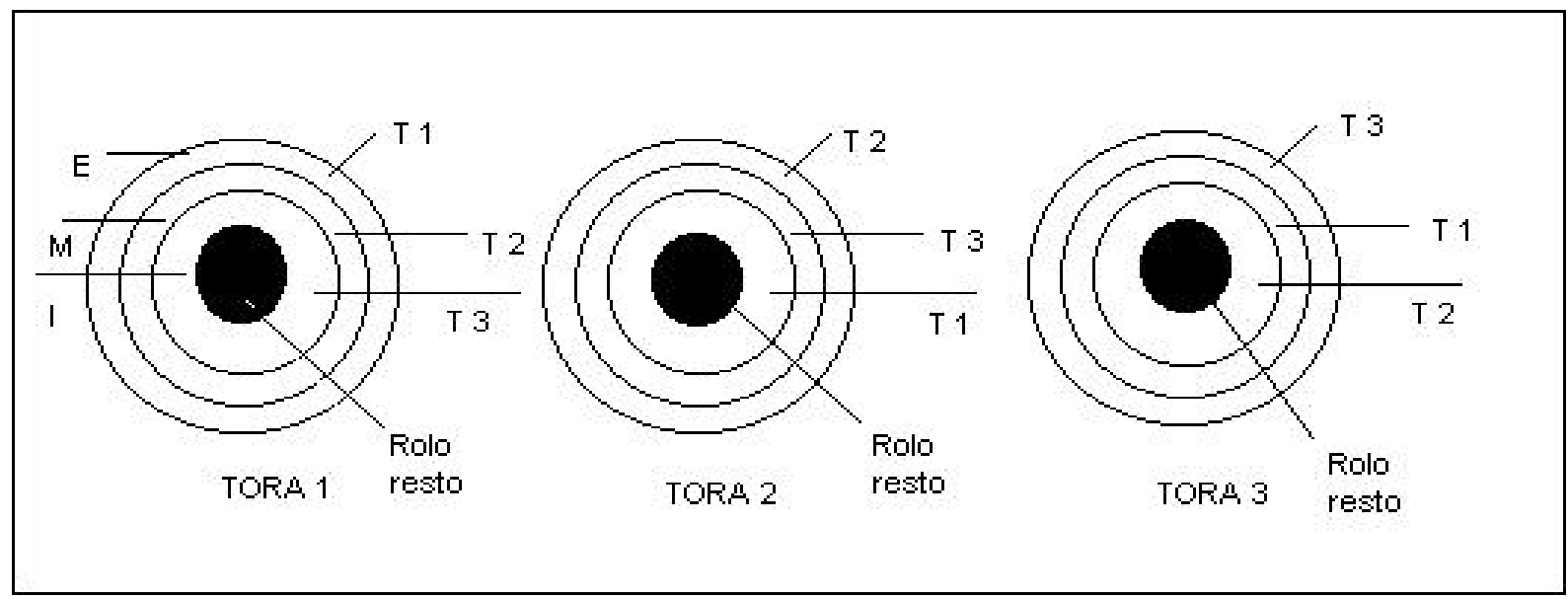

Figura 1 - Representação esquemática da distribuição das lâminas para os tratamentos T1, T2 e T3, nas seções externa (E), média (M) e interna (I) das toras.

Figure 1 -Schematic representation of the distribution of veneers for treatments T1, T2 and T3 in the external (E), medium (M) and internal (I) log sections.

O tratamento ocorreu em ambiente protegido com temperatura de $\pm 29^{\circ} \mathrm{C} \mathrm{e} \pm 80 \%$ de umidade relativa, para evitar a secagem acelerada das lâminas e promover a difusão do sal nos corpos-de-prova, nos tempos determinados. Logo após cada tempo para tratamento, as lâminas foram secas em uma secadora semi-industrial da marca Keller, a uma temperatura de $160{ }^{\circ} \mathrm{C}$ e uma velocidade de ar controlada para $1 \mathrm{~m}$ por minuto.

Sabe-se pela literatura (Williams \& Mauldin, 1986; Williams \& Amburgey, 1987; Barnes \& Williams, 1988a, b) que a madeira que contém $0,2 \%$ de EAB está protegida contra o ataque de Lyctus, com $0,3 \%$ está protegida contra cupins e que o nível de $0,5 \%$ a protege contra fungos de podridão-parda e de podridão-branca. Na pesquisa, optou-se pelo nível de retenção de $0,5 \%$ de EAB como o desejável.

\subsection{Determinação do Volume de Solução Aplicado nas Lâminas}

Para determinar o volume de solução e a quantidade de IA (EAB) a ser aplicado na madeira, necessita-se saber a massa seca de cada corpo-de-prova. Para isto, mediuse a densidade básica média (DB) da madeira utilizada no experimento, conforme a norma Copant (1971). Ao multiplicar o volume úmido de cada lâmina tratada pela densidade básica, obteve-se a massa seca (MS) para cada corpo-de-prova. O EAB é baseado nesta massa seca encontrada. Aplicando-se a relação entre bórax e ácido bórico, conforme o Quadro 1, obtém-se a quantidade de bórax na solução a ser aplicada. Determinando esta quantidade como $4 \%$, tem-se o volume de solução a ser aplicada. A quantidade de produto a ser retido é expressa em $\mathrm{kg}$ de $\mathrm{IA} / \mathrm{m}^{3}$ de madeira.

\subsection{Análise Química das Lâminas Tratadas}

Para as análises químicas de cada tratamento retiraram-se, ao acaso, nove lâminas, e de cada lâmina, aleatoriamente distribuídas, foram retiradas dez pequenas amostras de 2,5 $\times 2,5 \times 0,2 \mathrm{~cm}$, totalizando 90 corposde-prova por tratamento.

A determinação do boro baseou-se na metodologia proposta em Williams (1968), com análise espectrofotométrica $(555 \eta \mathrm{m})$. Utilizou-se um espectrofotômetro da marca Shimadzu modelo UV - 120-01.

\section{RESULTADOS E DISCUSSÃO}

\subsection{Determinação do Volume de Solução Aplicado nos Corpos-de-Prova}

O volume de solução e a quantidade de IA (\% EAB) a serem aplicados na madeira foram estabelecidos em função da massa seca de cada corpo-de-prova. Para isto, mediu-se a densidade básica da madeira utilizada,

R. Árvore, Viçosa-MG, v.27, n.3, p.321-326, 2003 
obtendo-se $0,31 \mathrm{~g} / \mathrm{cm}^{3}$. Ao multiplicar o volume úmido $\left(500 \mathrm{~cm}^{3}\right)$ de cada lâmina tratada pela densidade básica, determinou-se em $155 \mathrm{~g}$ a massa seca para cada corpode-prova. Da massa encontrada, $0,5 \%$ de EAB corresponde a $0,775 \mathrm{~g}$ de IA ou $1,55 \mathrm{~kg}$ de IA $/ \mathrm{m}^{3}$ de madeira.

Ao aplicar a relação entre o bórax e o ácido bórico (Quadro 1), foram obtidos 1,1935 g de bórax, o que equivale, para uma concentração de $4 \%$, a um volume de $29,8375 \mathrm{ml}$ de solução a ser aplicada em cada lâmina. Por questão de praticidade, o volume a ser aplicado foi de $30 \mathrm{ml}$.

Quadro 1 - Relação entre bórax e equivalente de ácido bórico (E AB)

Table 1 - Relation between borax and boric acid equivalent of $(B A E)$

\begin{tabular}{|l|c|c|}
\hline \multicolumn{1}{|c|}{ Solução } & Fórmula & Peso Molecular \\
\hline Bórax & $\mathrm{Na}_{2} \mathrm{~B}_{4} \mathrm{O}_{7} .10 \mathrm{H}_{2} \mathrm{O}$ & 381,37 \\
\hline $\begin{array}{l}\text { Equivalente ácido bórico } \\
\text { (EAB) }\end{array}$ & $4 \mathrm{H}_{3} \mathrm{BO}_{3}$ & 247,33 \\
\hline \multicolumn{3}{|c|}{ Relação peso/peso (bórax/EAB) $=1,54$} \\
\hline
\end{tabular}

\subsection{Análise Química Quantitativa}

Aplicaram-se os resultados da análise química na equação 1 , que apresentou um coeficiente de correlação $\left(\mathrm{R}^{2}\right)$ de 0,9948 , a fim de obter as correlações necessárias entre a leitura do espectrofotômetro e a quantidade de ácido bórico existente nas amostras de madeira.

$$
\mathrm{Y}=0,21393548+0,00898118 \mathrm{X}
$$

em que $\mathrm{Y}=$ leitura da densidade óptica (absorbância); e $\mathrm{X}=$ quantidade de ácido bórico nos corpos-de-prova.

No Quadro 2 estão as médias de retenção de boro (em \% EAB ou $\mathrm{kg}$ de $\mathrm{IA} / \mathrm{m}^{3}$ de madeira) para cada tratamento, com os respectivos desvios-padrão (DP) e coeficientes de variação (CV), além da comparação das médias pelo teste Tukey.

Como pode ser constatado no Quadro 2, não houve diferença significativa entre os tratamentos. Em termos gerais, os resultados obtidos foram satisfatórios ao considerar o objetivo proposto para o trabalho.

Embora não tenha havido diferença siginificativa, nota-se a tendência de diminuição do nível de retenção

R. Árvore, Viçosa-MG, v.27, n.3, p.321-326, 2003 $\left(\mathrm{kg} \mathrm{IA} / \mathrm{m}^{3}\right)$ com o aumento do tempo de difusão. Uma explicação para tal fato pode ser o teor de umidade das lâminas (Quadro 3). Uma peça de madeira com alta umidade tende a se equilibrar com o ambiente, ocorrendo uma movimentação da água livre do interior da madeira para as partes mais externas (Martins, 1988). Conseqüentemente, após o tempo de difusão e durante o processo de secagem, pode ocorrer uma migração do preservante para o exterior da peça, com uma perda dos compostos químicos que, porventura, se cristalizam na superfície da madeira (Cockcroft \& Levy, 1978). Segundo Morrel \& Freitag (1995), o teor de umidade diminui a retenção do boro com o decorrer do tempo de secagem, pois influi na redistribuição da água e do composto químico, ocasionando suas migrações para o exterior da madeira.

Quadro 2 - Retenção média de boro (\% de EAB) para os
tratamentose teste Tukey
$\begin{gathered}\text { Table } 2 \text { - Average boron retention ( } \% \text { BAE) for the } \\
\text { treatments and Tukey's test }\end{gathered}$
\begin{tabular}{|c|c|c|c|}
\hline $\begin{array}{c}\text { Tratamento } \\
\text { (minutos) }\end{array}$ & $\begin{array}{c}\% \mathrm{EAB} \\
\text { (DP) }\end{array}$ & $\begin{array}{c}\mathrm{CV} \\
(\%)\end{array}$ & $\mathrm{kg} \mathrm{IA} / \mathrm{m}^{3}$ \\
\hline 15 & $0,462 \mathrm{a} \pm 0,09$ & 19,48 & 1,43 \\
150 & $0,457 \mathrm{a} \pm 0,03$ & 6,56 & 1,41 \\
270 & $0,440 \mathrm{a} \pm 0,05$ & 11,36 & 1,36 \\
\hline
\end{tabular}

Letras iguais não diferem estatísticamente pelo teste Tukey $(\mathrm{p}<0,05)$.

Quadro 3 - Teor de umidade médio das lâminas para cada tratamento

Table 3 - Average moisture content of veneers for each treatment

\begin{tabular}{|c|c|}
\hline Tratamento (minutos) & Teor de Umidade (\%) \\
\hline 15 & $172,13 \mathrm{a}$ \\
150 & $167,29 \mathrm{a}$ \\
270 & $168,59 \mathrm{a}$ \\
\hline
\end{tabular}

Letras iguais não diferem estatísticamente pelo teste Tukey $(\mathrm{p}<0,05)$.

O manuseio das lâminas pode provocar alguma perda, principalmente dos sais cristalizados na superfície. Em relação aos resultados obtidos, não foi possível definir em qual momento houve esta perda, embora seja mais provável que tenha ocorrido durante a secagem.

Nos testes desenvolvidos, os teores de umidade contidos nas lâminas no momento da aplicação do bórax não diferiram estatisticamente entre si e superaram em 
muito os $100 \%$ (Quadro 3). Esta característica se apresenta como o principal fator para a falta de diferenças significativas entre os tratamentos.

Constatou-se que, logo após 15 minutos de aplicação de bórax, podem ser obtidas uma difusão e uma retenção do produto satisfatórias em função do alto teor de umidade das lâminas.

Além da melhor retenção, a escolha do melhor tratamento (em relação ao tempo de difusão) e do momento em que deverá ser aplicado o produto preservativo irá depender das características de cada linha de produção.

\section{REFERÊNCIAS BIBLIOGRÁFICAS}

AMBURGEY, T. L. The need for co-biocides when treating wood with borates. In: INTERNACIONAL CONFERENCE ON WOOD PROTECTION WITH DIFFUSIBLE

PRESERVATIVES, 1., 1990, Proceedings... 1990. p. 51-52.

BARNES, H. M.; WILLIAMS, L. H. Integrated protection against lyctid beetle infestations. V. Selecting efficient schedules for pressure treatment of tropical hardwood lumber with polyborates. Forest Product Journal, v. 38, n. 9, p. 13-19, 1988a.

BARNES, H. M.; WILLIAMS, L. H. Integrated protection against lyctid beetle infestations. VI. Thermal treatment of tropical hardwood lumber with polyborates. Forest Product Journal, v. 38, n. 9, p. 20-21, 1988b.

BECKER, G. Treatment of wood by diffusion of salts. Stockolm, The Internacional Resource Group on Wood Preservation, 1976. 9 p. (Document IRG, 368)

CARR, D. R. Timborised timber. London: Borax Consolidated Limited, Borax house, 1958a. 40 p.

CARR, D.R. Boron as a timber preservative. Part I. Wood, n. 9 , p. $380-382,1958$ b.

CHUDNOFF, M. Tropical timber of the world. Forest Products Laboratory, Forest Service, 1980. 831 p.

COCKCROFT, R.; LEVY, J. F. Bibliography on the use of boron compounds in the preservation of wood. Journal of The Institute of Wood Science, v. 13, n. 8, p. 28-30, 1973.

COMISION PANAMERICANA DE NORMAS TECNICAS - COPANT. Método de determinacion del peso específico aparente. 1972. 4 p. (Proyecto COPANT, v. 30)
DÉON, G. Manual de preservação das madeiras tropicais em clima tropical. Paris: ITTO-CIRAD, 1986. 115 p. (Série Técnica ,3)

HUMMEL, A. C. et al. Diagnóstico do subsetor madeireiro do estado do Amazonas. Manaus: SEBRAE, 1993. 54 p.

INSTITUTO BRASILEIRO DE DESENVOLVIMENTO FLORESTAL - IBDF. Norma de controle de qualidade e classificação de compensados. Brasília: 1985. 79 p.

LAKS, P. E. et al. Evaluation of adhesives for bonding borate-treated flakeboards. Forest Products Journal, v. 38, n. 11/12, p. 23-24, 1988.

LOUREIRO, A. A.; SILVA, M. F.; ALENCAR, J. C. Essências madeireiras da Amazônia. Vol. II . Manaus: CNPq-INPA, 1979. v. 2, p. 137-139.

MARTINS, V. A. Secagem de madeira serrada. Brasília: IBDF/DPq - LPF, 1988. 56 p.

MORREL, J. J.; FREITAG, C. M. Effect of wood moisture content on diffusion of boron-based biocides through Douglas-fir and Western Hemlock lumber. Forest Products Journal, v. 45, n. 3, p. 51-55, 1995.

MORREL, J. J.; SEXTON,C. M.; PRESTON, A. F. Effect of moisture content of Douglas-fir heartwood on longitudinal diffusion of boron from fused borate rods. Forest Products Journal, v. 40, n. 4, p. 37-40, 1990.

PUETTMANN, M. E.; SCHMIDT, E. L. Boron diffusion treatment of aspen lumber stored under various relative humidities. Forest Products Journal, v. 47, n. 10, p. 47$50,1997$.

VIANEZ, B. F. Aspects of boron diffusion through hardwoods. Wales: University of Wales, 1993. 244 p.

VIDEN, P. Treatment with wood in the 1990s. In: INTERNATIONAL CONFERENCE ON WOOD PROTECTION WITH DIFFUSIBLE PRESERVATIVES, 1., 1990, Proceedings... 1990. p. 22-25.

WILLIAMS, A. I. The extraction and determination of dissodiun octaborate in Sitka Spruce. The Analyst, v. 99, n. 2, p. 111-115, 1968.

WILLIAMS, L. H.; AMBURGEY, T. L. Integrated protection against lyctid beetle infestations. IV. Resistence of boron-treated wood (Virola spp.) to insect and fungal attack. Forest Products Journal, v. 37, n. 2, p. 10-17, 1987.

R. Árvore, Viçosa-MG, v.27, n.3, p.321-326, 2003 
WILLIAMS, L. H.; MAULDIN, J. K. Integrated protection against Lyctid Beetle infestations. III. Implementing boron treatement of Virola lumber in Brazil. Forest Products Journal, v. 36, n. 11/12, p. 24-28, 1986.
ZAVALA, Z. D. Diagnóstico de la industria de tableros contrachapados en el área del D.F. Revista Ciencia Forestal en México, v. 15, n. 68, p. 61-83, 1990a. 